\title{
Interactive teaching workshop for foundation year doctors at a district general hospital
}

\author{
Authors: Carmen Lau, Helen Parry, Julia Zhu, Motahare Yadegarfar and Natali Vigneswaran
}

\section{Aims}

To assess whether teaching skills training for foundation-year (FY) doctors improves confidence in teaching.

\section{Methods}

In November 2017, five clinical education fellows (CEFs) ran two identical 2-hour teaching workshops for a separate group of FY doctors in the evening. All of the CEFs involved have undertaken formal teaching skills training at a higher education institution.

Each workshop was limited to a maximum of 24 participants. The workshop consisted of a 10-minute introductory session on planning a teaching event. This was followed by four 20-minute stations, with participants split into groups of six. Stations were delivered on the topics of small group teaching, teaching on the job, how to give feedback and dealing with the challenging learner. Participants filled in anonymous pre- and post-course questionnaires related to their subjective confidence levels on different teaching domains. Confidence levels were scored using a 1-5 Likert scale with a value of 1 assigned to 'not at all confident' and a value of 5 assigned to 'very confident'.

\section{Results}

There were 23 and 20 participants on 15 November 2017 and 22 November 2017 respectively. The median confidence scores for preand post-course questionnaires were calculated for each teaching domain. There was a global increase in median confidence levels in all domains. The greatest increase in median confidence score was found in the following stations: teaching while on call, teaching small groups and dealing with challenging learners (Table 1).

All of the participants gave positive overall feedback on the workshop. Participants found the stations on dealing with challenging learners, giving feedback and planning a teaching session particularly useful. It was commented on that the workshop also increased motivation to teach.

Author: Medical Education, South Warwickshire NHS Foundation Trust, Warwick, UK

\begin{tabular}{lll} 
Table 1. Summary of results & \multicolumn{2}{l}{ Median scores } \\
\cline { 2 - 3 } Topics & Pre & Post \\
& 3 & 4 \\
Planning a teaching session & 3 & 4 \\
Teaching on the wards & 2 & 4 \\
Teaching while on call & 3 & 5 \\
Teaching small groups (up to eight students) & 3 & 4 \\
Using audio-visual aids effectively & 3 & 4 \\
Providing feedback & 3 & 4 \\
Adapting your teaching style based on the level & 3 & \\
of training of your students & & 4 \\
Dealing with challenging learners & 2 & 4
\end{tabular}

\section{Conclusion}

The workshop attracted a lot of interest from FY doctors, which demonstrates a gap in current teaching training provided as part of the curriculum. The timing and local proximity of the workshop enabled participants to attend around their clinical commitments. Following the workshop, questionnaire data highlighted that participants were more confident and motivated to teach. FY doctors also expressed an interest in undertaking further training in other topics such as learning theories. Overall, our questionnaire data supports the use of this workshop as an adjunct to the professional development of FY doctors.

Despite all efforts, some participants had to cancel their attendance last minute due to clinical workload. Further to this, due to the time constraints of a 2-hour session, the scope of content was limited to only key concepts. This demonstrates that consideration is needed to include substantial teaching training as part of FY protected teaching to ensure that clinical workload does not negatively impact on learning opportunities. The authors hope to further develop this workshop by organising a teaching programme consisting of a series of workshops and incorporating practical teaching skills training into FY doctors protected teaching time in the future.

\section{Conflict of interest statement}

No conflict of interest. 